\title{
EDITORIAL
}

\section{Domestic Service Robots in the Real World}

\author{
Luca Iocchi • Javier Ruiz-del-Solar • Tijn van der Zant
}

Published online: 17 August 2011

(C) Springer Science+Business Media B.V. 2011

\section{Introduction}

The use of robots in domestic environments has increased largely in the recent years. There are already several millions robots used for basic household chores (e.g. vacuum cleaning) or for entertainment. It is expected that domestic service robots become more and more useful, autonomous, multipurpose and reliable, and that they will be able to interact with humans and objects in the real world in a natural way. This challenge poses a large number of unsolved problems across many scientific disciplines.

The aim of this special issue is to present recent advances in domestic service robotics, showing robots that are able to operate in real home

L. Iocchi

Dept. of Computer and Systems Science,

University of Rome "a Sapienza”, Rome, Italy

J. Ruiz-del-Solar ( $\square)$

Dept. of Electrical Engineering \& Advanced Mining

Technology Center, Universidad de Chile,

Santiago, Chile

e-mail: jruizd@ing.uchile.cl

T. van der Zant

Dept. of Artificial Intelligence,

University of Groningen, Groningen,

The Netherlands environments autonomously. The home environment is defined as 'any place where people live in their daily lives', which can include, for example, a living room, a kitchen or a garden. The focus is in different modes of natural interaction with humans, real-world high-level cognition, manipulation of domestic objects, useful applications, cost-effectiveness of the solution, the effect of appearance on acceptance and more.

The use of competitions for benchmarking specific functionalities and integrated systems is very common. In the field of domestic service robotics, RoboCup@Home [1] is the largest initiative in terms of both the number of participating teams and the amount of research issues taken into account. The goal of RoboCup@Home is to foster the development of versatile domestic service robots, that can operate safely in all situations that people encounter in daily life, through a dynamical form of benchmarking formed by many different tests where many functionalities are tested at the same time in an integrated way. Therefore, good results are obtained only if a complete system implementing all the desired abilities is demonstrated to work during the competition.

In this special issue, special attention was paid to submissions where the robot capabilities were validated in a real-world setting such as the RoboCup@Home benchmark or during long lasting experiments in the real-world. 
This special issue received fourteen highquality submissions. From them five were selected to be included in this special issue. The main topics addresses by these submissions are: cooperative human-robot interaction, manipulation of domestic objects, multimodal human-robot interaction, human detection and identification in uncontrolled setups, and high-level cognition in domestic environments.

Nakamura et al. define extended mobile manipulation as a process in which a robot is able to manipulate an object through speech interaction with a human. They propose a method for learning novel objects from audio-visual input. A voice conversion technique allows the robot to pronounce the acquired out-of-vocabulary word intelligibly. They implemented a robotic system that carries out interactive mobile manipulation tasks using the proposed method.

Rosenthal, Veloso and Dey analyze the idea that a robot should proactively ask humans for help in order to solve complex situations and overcome some of its limitations. They present an extended interaction model that includes the availability of humans in the environment, and demonstrate how a navigation planner can incorporate this information to plan paths that increase the likelihood that a robot can find an available helper when it needs one.

Correa et al. propose a human detection and identification system that uses thermal and visual information sources. Both sources are used in order to detect human body and faces, and human identification is obtained by analyzing the face information. Active vision mechanisms improve the relative pose of candidate objects/persons in case direct identification is not possible. The results show the convenience of using thermal and visual information in order to get robust detection and identification in real domestic environments.

Breuer et al. describe the main aspects of the control and software architecture of a versatile autonomous domestic robot. The control architecture is able to cope with various and changing robot tasks, while the software architecture supports the rapid integration of new functionalities. They also report novel and robust algorithms centered around multi-modal human robot interaction, semantic scene understanding and SLAM, which are validated in real domestic applications.

Schiffer, Ferrein and Lakemeyer present the integration of qualitative representations and reasoning for positional information into high-level robot control. They propose a framework that uses Readylog, a special robot programming and planning language, and fuzzy set based qualitative concepts with positional fluents and contexts. Reported experiments demonstrate how qualitative positional fluents based on a fuzzy set semantics can be deployed in domestic domains, and showcase how reasoning with these qualitative notions can seamlessly be applied in complex robot tasks.

Four of the special issue articles validate their methodologies using standard tests of the RoboCup@Home benchmark. Taking into account this, as well as the importance of using benchmarks in domestic service robotics, we will describe the RoboCup@Home benchmark and some of the main results of its application in the last years.

\section{Domestic Service Robots Benchmarks}

Benchmarking has many advantages, such as the creation of standard references and metrics. However, there is also a possible disadvantage when benchmarking remains static over time, that is the progress of solutions towards a local optimum, without having a guarantee that the devised solution will work in general. In robotics this can be a huge problem. There are many examples where robots that operate very well in the laboratory and obtain good results in some specific benchmarking activity, do not function so well in the real world. Having dynamic benchmarks that test integrated systems (rather than single functionalities) and that change over time, ensures high quality and robust scientific solutions, while specialized solutions looking for a local optimum will not be rewarded in the long term.

RoboCup@Home adheres to dynamic benchmarking of integrating systems, by devising complex tests and by changing most of them on a yearly basis. The versatility thus stimulates teams 
to create general solutions, which have a higher chance to work in many different settings than with the use of a benchmark which does not change. Since the goal of RoboCup@Home is to foster research for the creation of a general purpose domestic service robot, the robot has to be flexible in many aspects.

The downside of the changing tests is that this co-evolutionary process does not guarantee progress into the desired direction of versatile autonomous domestic service robots. It is essential that scientific standards are applied to this type of flexible benchmarking. In RoboCup@Home statistical analysis of the outcome of the competition is used to steer the league into interesting directions (see [1] for details).

We firmly believe that this approach to benchmarking will quickly raise the general level of research groups developing solutions in domestic service robotics and may even fill the gap between laboratory experimental settings and real robotic applications, thus providing an important link with industries. The statistical benchmarking approach is the only viable approach for the benchmarking of real-world robots. Any form of fixed benchmarking will only create local optimal solutions that are unlikely to work in the real world. This approach could be effectively ported to other robotic application fields where the environment has a high level of uncertainty, such as the fields of rescue, space and reconnaissance robots.

Statistical analysis of RoboCup@Home benchmarking The functional abilities that have been considered in RoboCup@Home so far are: Navigation, Mapping, Person Recognition, Person Tracking, Object Recognition, Object Manipulation, Speech Recognition, Gesture Recognition, Cognition (since 2010). The score system allows to relate score points to functional abilities in each tests, thus we can analyze the amount of score available and those actually gained by the best teams for each functionality in each test. In this way, we are able to evaluate the average increase of performance in the given skills over years even when changing the tests, in order to make them closer to real world applications.
Table 1 Achieved scores for the desired abilities

\begin{tabular}{llll}
\hline Ability & $2008[\%]$ & $2009[\%]$ & $2010[\%]$ \\
\hline Navigation & $40 / 25$ & $47 / 40$ & $33 / 20$ \\
Mapping & $100 / 44$ & $100 / 92$ & $21 / 10$ \\
Person recognition & $32 / 15$ & $69 / 37$ & $57 / 23$ \\
Person tracking & $100 / 81$ & $100 / 69$ & $100 / 72$ \\
Object recognition & $29 / 8$ & $39 / 23$ & $6 / 1$ \\
Object manipulation & $3 / 1$ & $48 / 23$ & $29 / 8$ \\
Speech recognition & $87 / 37$ & $89 / 71$ & $50 / 38$ \\
Gesture recognition & $0 / 0$ & $0 / 0$ & $62 / 26$ \\
Cognition & - & - & $17 / 3$ \\
Average & $41 / 21$ & $61.5 / 44.4$ & $41.6 / 22.4$ \\
\hline
\end{tabular}

Table 1 presents the percentage of the available scores actually gained by the teams during RoboCup@Home 2008, 2009 and 2010 competitions, related to each of the desired abilities. The first value is the maximum score achieved by some team and the second value is the average score achieved by the finalist teams. This table allows for many considerations, such as: 1) which abilities have been mostly successfully implemented by the teams; 2) how difficult are the tests with respect to such abilities; 3) which tests and abilities need to be changed in order to guide future development into desired directions.

By analyzing the results of team performance it is possible to decide about future development of the benchmarks. Possible adjustments are: 1) increasing the difficulty if the average performance is high; 2) merging of abilities into high-level skills, more realistic tasks; 3) keeping or even decreasing difficulty if the observed performance is not satisfying; 4) introducing new abilities and tests.

Other important parameters to assess the success of a benchmark are the number of participating research groups (teams) and the general increase of performance over the years. In Table 2, the number of teams participating to the

Table 2 Measures indicating general increase of performance

\begin{tabular}{llllll}
\hline Measure & 2006 & 2007 & 2008 & 2009 & 2010 \\
\hline Number of teams & 12 & 11 & 14 & 18 & 24 \\
Total amount of tests & 66 & 76 & 86 & 127 & 164 \\
Percentage of succ. tests & $17 \%$ & $36 \%$ & $59 \%$ & $83 \%$ & $74 \%$ \\
Avg. succ. tests p. team & 1.0 & 2.5 & 4.9 & 7.3 & 6.3 \\
\hline
\end{tabular}


international competition is shown in the first row. The second row shows the increase in the total number of tests executed by all the teams during the competition. The execution of over 100 tests since 2009 and some of them outside the field (i.e., in a real environment) in 2010 confirms the significance of the statistical analysis we are performing. The third row contains the percentage of successful tests, i.e., tests where some score greater than zero was achieved, showing a significant and constant increase in the years compared also to the general increase of the difficulty of the tests. Finally, the fourth row contains the average number of successful tests for each team. This is a very important measure, since the enormous increase from 1.0 tests in 2006 to 7.3 in 2009 (and 6.3 in 2010 with more difficult tests) is a strong indication for an average increase in robot abilities and in overall system integration. A team successfully participating in an average of 7 tests (that are quite different each other) demonstrates not only effective solutions and implementation of all the desired abilities, but also a flexible integrated system that has important features for real world applications.

The results obtained by the analysis reported here clearly show that the RoboCup@Home methodology of dynamic benchmarking is producing a quick and significant progress in domestic service robotics.

\section{Reference}

1. Wisspeintner, T., van der Zant, T., Iocchi, L., Schiffer, S.: RoboCup@Home: scientific competition and benchmarking for domestic service robots. Interaction Studies 10(3), 393-428 (2009) 